\title{
PERRY, PERSONAL IDENTITY AND THE “CHARACTERISTIC” WAY
}

\section{NATHAN OAKLANDER}

Some who attempt to analyse or define personal identity solely in terms of memory are making two claims about persons. First, they are claiming that a person is essentially non-bodily. More specifically, they say that a person is a temporally ex tended totality (series) of mental states or experiences connected in a certain way. Second, they are claiming that the relation that connects all the mental states that are members of the same person is memory. The idea is that A and B are states of the same person if and only if A contains, or would contain under given conditions, a memory of some experience which is an element in B. ${ }^{1}$ Others who appeal to memory to understand personal identity claim that a suitable body (or brain) is necessary for memory and thus that a person is essentially a bodily thing. ${ }^{2}$ One of the most serious objections to both versions of the memory theory is Butler's claim that "memory presupposes, and so cannot constitute, personal identity". ${ }^{3}$ In a recent paper John Perry has attempted to vindicate the memory theorist from the charge of circularity. ${ }^{4}$ I shall argue, however, that either Perry does not avoid the problem of circularity, or he avoids it as the cost of giving an analysis of memory that is inadequate as an account of personal identity. I shall proceed by first explaining what is involved in calling the memory theory "circular" and by then criticising Perry's attempt to avoid that allegation.

1 See H.P. Grice, "Personal Identity", Mind 50, No. 200 (October 1941), 330-350. Reprinted in Personal Identity, J. Perry (ed.) (Berkeley: University of California, 1975); hereafter referred to as PI. A. Quinton, "The Soul", Journal of Philosophy 59, No. 15 (July 19, 1962), 393-409. Reprinted in PI.

2 See D. Wiggins, Identity and Spatio-Temporal Continuity (Oxford: Basil Blackwell, 1971) 42-57; D. Wiggins, "Locke, Butler and the Stream of Consciousness", in The Identities of Persons, A.O. Rorty (ed.) (Berkeley: University of California, 1976); 139-173. D. Parfit, "Personal Identity", Philosophical Review 80, No. 1 (January 1971), 3-27 and reprinted in PI; D. Parfit, "Lewis, Perry, and What Matters", in The Identities of Persons, op. cit, , 101-02. To say that a person is essentially material does not imply that persons are definable in terms of the concepts of the sciences of matter, but that they are beings that are 'essentially enmattered', D. Wiggins, "Locke, Butler and the Stream of Consciousness", op. cit., 152.

3 J. Butler, "Of Personal Identity", reprinted in PI.

4 J. Perry, "Personal Identity, Memory, and the Problem of Circularity", in PI, 135155. For other attempts see S. Shoemaker, "Persons and Their Pasts", American Philosophical Quarterly, Vol. 7 No. 4 (October, 1920), pp. 269-285. D. Parfit, "Personal Indetity",op. cit., and David Wiggins, Sameness and Substance, (Oxford: Basil Blackwell, 1981). Shoemaker's way out is effectively criticized in H. Noonan, Objects and Identity, (The Hague: Martinus Nijhoff, 1980), Chapter 14. 
According to Perry and many others, the problem of personal identity centres around the following question: What makes an experience, that is, a thought, emotion, belief, or event, mine? Alternatively, what is the basis for saying that two or more experiences, for example, the belief in 1969 that Nixon is president, and the belief in 1979 that Carter is president, are experiences of one and the same person ${ }^{5}$ The initial response of the memory theorist is that an experience is mine if and only if I can remember it. So stated the memory theory is inadequate because I can remember somebody else's experience and when I do the other person's experience does not become my experience. For example, suppose that I seem to remember turning off the stove this morning, when in fact not $I$, but my friend turned off the stove. In such a case, if we allow that any kind of memory, even apparent memory, is necessary and sufficient for personal identity, then I would be identical with my friend and that is absurd.

The natural and immediate response to the above objection is to modify the memory theory by claiming that not any kind of memory is necessary and sufficient for personal identity, real memory is required. The absurdity just mentioned does not follow because I do not really remember turning off the stove, I only seem to remember. The adequacy of this reply requires an account of the difference between real memory and apparent memory, and it is at this stage that the circularity of the memory theory emerges. For what is it to really remember something? Clearly, it involves seeming to remember plus something else, but what else? Is it that I really remember doing $\mathrm{X}$ when I seem to remember doing $\mathrm{X}$ and actually did do $X$ ? If so, then since to say that ' $I$ actually did do $X$ ' is tantamount to saying that ' $I$ am now identical with the person who earlier did $X$ ', it follows that a necessary condition of real memory is personal identity. Thus it is argued that one cannot analyse personal identity in terms of real memory without falling into circularity. ${ }^{6}$

Perry attempts to save the memory theorists from circularity by showing that the concept of "the same person" need not be included in a correct analysis of real memory (memory, for short). There are three crucial elements in Perry's analysis of memory which I shall first state and then discuss. He says that,

5 See, for example, J. Perry, Introduction to PI, 9-11. A.J. Ayer, The Central Questions of Philosophy, (New York: William Morrow, 1974), 114-117; C.D. Broad, The Mind and Its Place in Nature, (London: Routledge \& Kegan Paul, 1925), 536-557; A.C. Ewing, The Fundamental Questions in Philosophy, (New York: Collier Books, 1962), 120-125.

6 See J. Perry, A Dialogue on Personal Identity and Immortality, (Indianapolis: Hackett Pub., 1978), 21-31, and J. Perry "Personal Identity, Memory, and the Problem of Circularity", op. cit., 137-143. 
A remembers $e$ if and only if

(1) A represents the past occurrence of an event of some type E;

(2) B witnessed e;

(3) B's witnessing of $\mathrm{e}$ is M-related to A's representation of the past occurrence of an event of type E. ${ }^{7}$

The first condition he calls the Representation Condition. At the time we remember an event we always indicate the past event in some way or another, e.g., we give a verbal description of it, we paint a picture of it, or we produce a mental image of it. The second condition he calls the Weak Witnessing Condition. Initially, Perry claims that A and B are live human bodies, although it is not part of the (weak) witnessing condition either that $A$ and $B$ are identical or that they are bodies belonging to the same person. However, he later claims that A and B may stand for, "not just human bodies but human bodies and any other sorts of things, ghosts or even gorse-bushes...", thus implying that the witnessing condition does not necessarily involve a human body (or brain). The third condition which he calls the Linking Condition is needed to ensure that the past witnessing leads to the present representing in the characteristic way. But what exactly is the characteristic way in which a witnessing leads to a representing? How are we to identify the M-relation? The answer to these questions are absolutely crucial for unless Perry can cast some intellectual light on the M-relation, the distinction between really remembering and seeming to remember will remain obscure and the problem of circularity will remain unresolved.

To bring the central issue into sharper focus let us consider three situations that Perry describes that give rise to the need for the linking condition or the M-relation. First, Smith examines a green cube, and later vividly describes his examination of it. Second, Hennig examines a green cube, then receives an electrical shock that wipes out his memory. The Electric Company, in compensation, has him hypnotized and told that when he awakes he will remember examining a green cube. Third, Cohen, when a young boy, knocked over a Menorah so that the candles fell into and spoiled a tureen of soup. Since it happened so long ago he forgot it, but hearing his parents talk about the story he later comes to remember doing it. ${ }^{9}$ Perry maintains that Smith is really remembering a past experience whereas both Hennig and Cohen are not really remembering, but only seeming to remember a past experience. And here we must ask, What then is the difference between the Smith case on the one hand and the Hennig and Cohen cases on the other? Perry's answer is that in the Smith case the M-relation is present whereas in the other cases it is not.

7 J. Perry, "Personal Identity, Memory, and the Problem of Circularity", op. cit., 146; emphasis added.

8 Ibid., 147.

9 The first two cases are from J. Perry, "Personal Identity, Memory, and the Problem of Circularity", op. cit., 139 and 146. The third is from A Dialogue On Personal Identity and Immortality, op. cit., 131. 
The point to note is that an appeal to the M-relation is legitimate and useful only if he can identify the M-relation independently of the concept of memory. Thus, we must turn to the crucial question: What is the M-relation?

Perry begins to identify the M-relation by stating his agreement with the view of Martin and Deutscher, ${ }^{10}$ that the relation is causal. Of course, the $M$ relation cannot simply be identified with any causal link between a witnessing and a representing since as Perry says, "the witnessing must not just cause the representation, it must cause it in a certain way". " What then is the characteristic way in which a witnessing causes a representing? Perry denies that the characteristic causal link could be identified with anything the scientist tells us concerning the mechanism involved in memory:

Scientists are trying to discover the causal mechanisms involved in memory. Suppose that they discover that a certain process is involved in memory. Could our linking condition be that that process led from B's witnessing to A's representing? No ....12

Positively, Perry attempts to identify the M-relation by introducing the technical term "recollection" which he defines stipulatively as follows:

$A$ recollects $e$ if and only if

(1) A represents the past occurrence of an event of type E;

(2) B witnessed e, and e is of type E;

(3) $\mathrm{B}$ and $\mathrm{A}$ are the same live human body. ${ }^{13}$

Curiously, even though recollection is introduced to help us understand the M-relation and ultimately the concept of memory, Perry says that,

recollection is a significantly different notion from memory. Returning to the case of the green cube, both Smith and Hennig recollect examining the cube, though only Smith remembers. ${ }^{14}$

But this is very puzzling, for if we can recollect without remembering, that is, without the representing being caused in the right way by the witnessing, then how can recollection help us to understand the characteristic causal link or the M-relation?

Perry's answer is found in the following passage which I shall quote at length:

10 C.B. Martin and M. Deutscher, "Remembering", Philosophical Review 85, No. 2 (July, 1966).

11 J. Perry, "Personal Identity, Memory, and the Problem of Circularity", op. cit., 146.

12 Ibid., p. 146.

13 Ibid., 147; emphasis added.

14 Ibid., 148; emphasis added. 
PERRY, PERSONAL IDENTITY AND THE “CHARACTERISTIC" WAY 39

An unaided case of recollection is one in which the representing of $A$ is not explained by provision of information about $e$ other than B's witnessing of it. Now any ordinary human is drawn to the belief that there is an explanation for the frequent occurrence of unaided cases of recollection, that there is some process, material or immaterial, gross or sublime, complex or simple, which frequently occurs when a human being witnesses an event and leads to that same human's later representation of it. When the witnessing of an event leads by this process to a later representation of it, the witnessing and the representation are $\mathrm{M}$-related. ${ }^{15}$

According to Perry this way of identifying the M-relation removes the circularity from (1) his analysis of memory and (2) the memory theorists analysis of personal identity. For (1) the M-relation is not identified in terms of memory, but rather as the relation that explains the great many cases of recollection, and (2) he can now understand the claim that "a mental state $\mathrm{A}$ contains a memory of some experience in a mental state $B$ " in terms of the three conditions of memory stated above (p. 3). Thus, since nowhere in the analysis of memory need we assume that the mental states $A$ and $B$ belong to the same person, the alleged circularity of the memory analysis of personal identity is avoided.

In spite of the care in which Perry attempts to identify the M-relation it seems to me that he never succeeds in making it intellectually visible. If, as Perry says, the $M$-relation is a process that may be 'simple or complex, material or immaterial, gross or sublime', then it may be anything, and so is something we know not what. His description of the M-relation is too general, it does not enable us to single out before our minds the object such a relation might be. Furthermore, there are no criteria the presence of which are logically sufficient or necessary for the existence of the M-relation. We cannot infer from the sameness of body $A$ and body $B$ that the $M$-relation obtains because Perry distinguishes the $\mathrm{M}$-relation and the relation of being or belonging to the same human body. Indeed, he says that,

by virtue of our lack of knowledge of the nature of the M-relation, it becomes possible to think of the two as separate; we are able to imagine the possibility that certain witnessings and representings might be $M$ related, though not experiences of the same human body. ${ }^{16}$

Nor can we detect the presence of the M-relation by an appeal to a similarity of psychological characteristics between the witnesser and the representer. For in the Cohen case we have a person whose personality has presumably exhibited a continuity over time, but by hypothesis he is not M-related to the events that he previously experienced. Thus, the similarity of psychological traits is not sufficient to guarantee the presence of the M-relation. It appears

15 Ibid., 148.

16 Ibid., 149; emphasis added. 
then, that there is no principle by which we can identify the presence of absence of the M-relation other then to say that it is the relation that obtains when we really remember. But then, we have employed the concept we are trying to understand, thus falling into circularity.

Perhaps the circularity or inadequacy of Perry's account of real memory can be seen more clearly if we return to his attempt to identify the M-relation. Recall, that in order to identify the M-relation Perry appeals to the distinction between aided and unaided recollection, and claims that the M-relation is the process that explains cases of unaided recollection. But exactly how are we to understand the distinction between aided and unaided recollection? I do not think that Perry can provide an adequate non-circular answer to that question. Consider the following cases: Smith examines a green cube and later he sees a green square that causes him to represent the earlier experience. Smith sees Gone with the Wind and later hears some people talking about Clark Gable and, as a result, represents the past event. Finally, Smith hears a recording of Beethoven's Fifth Symphony and later hears some notes that cause him to represent it. The three cases I have described are familiar to all of us. We witness an event and later we become aware of an object or state of affairs that "triggers" our representing of it. Given that the same body does the witnessing and the representing, are these cases of aided or unaided recollection? Does the witnessing cause the representing in the characteristic way or not? Is the M-relation present or absent? The very fact that it is not clear how we should answer these questions casts doubt on the hope to independently identify the M-relation by an appeal to aided and unaided recollection. The doubt is reinforced by considering the possible answers to the questions just raised.

Suppose we say that in the cases just described Smith's recollections are unaided and that, therefore, the M-relation is present. Surely this is a plausible view to take since we want to say not only that Smith recollects past events, but that he remembers them, and on Perry's view memory requires the Mrelation. However, if Smith's recollections are unaided, then what grounds are there for saying that Cohen's recollection of knocking over the Menorah, and Hennig's recollection of examining a green cube, are cases of aided recollection? In all these cases the representing is "triggered" by an external object or state of affairs that the recollector is aware of before the representing. Thus, there appears to be no relevant difference between Smith's unaided recollections on the one hand and Cohen's and Hennig's aided recollections on the other, unless we say that Smith really remembers whereas Cohen and Hennig only seem to remember.

As that move lands us back in a circle, suppose we say that Smith's recollections are aided and that therefore, the M-relation is not present. Then, since the M-relation is necessary for memory it would follow that Smith does not really remember examining the green cube, seeing Gone with the Wind, or hearing Beethoven's Fif th Symphony. That consequence is, however, extremely implausible if not absurd. Consequently, either Smith's recollections are 
unaided and Perry's analysis of memory is circular, or the M-relation is not necessary for memory and Perry's analysis of memory is inadequate.

The objection that I am making against the causal theory of memory and the causal theory of personal identity that is based upon it is that it makes an appeal to an "appropriate" or "characteristic" causal process that is just as mysterious and as much in need of philosophical understanding as is the relation that unites different experiences of the same person. Clearly, it is of no use to identify the M-relation as that relation that obtains when the witnessing and the representing are experiences belonging to the same person, for then Butler's charge would be justified after all. Fortunately, Perry realizes this and puts a wedge between the M-relation and identity. Unfortunately, by allowing that two experiences of the same person might not be $\mathrm{M}$ related, he avoids the circularity of the memory theory by embracing an inadequate analysis of personal identity. To see what is involved in this last point note first that an adequate analysis of personal identity requires that the analysans and the analysandum are logically equivalent. Yet that condition is not Perry's analysis. For in a situation previously described Hennig is not $\mathrm{M}$-related to a past witnessing, and hence he does not remember the previously witnessed event, al though ex hypothesi he retains his personal identity. Thus, on the assumption that personal identity is to be analysed in terms of memory, it follows that Hennig is and is not the same person who examined the green cubes. Since that consequence is contradictory, the memory theory of personal identity from which it is derived must be rejected.

In response to the above argument, one may claim that not actual memory, but only possible memory is necessary for personal identity. Indeed, Perry does say that "a sufficient and necessary condition of my having participated in a past event is that I am able to remember it". ${ }^{17}$ However, I am not sure that the appeal to possible memory or the ability to remember removes the problem of the Hennig case. For Hennig has his memory wiped out and therefore it is not clear that he does have the ability to remember examining the green cubes or anything else. How can there be a series of actual or possible person stages linked by the M-relation when, by hypothesis, all Hennig's memory links to the past (including the immediately preceding stages) have been severed? It would appear therefore, that Perry's analysis avoids circularity at the cost of embracing an inadequate view of personal identity.

What advantages then does the memory theory of personal identity have over the view that in cases of personal identity there is a simple unanalysable relation that unites all of a series of expereinces into the experiences of one person? And why is it preferable to the substantialist view recently defended by Chisholm that there is a single persistent substantial self that is the support of all the experiences that belong to one person. ${ }^{18}$ As Perry states the 17 J. Perry, "The Importance of Being Identical", in The Identities of Persons, op. cit., 69.

18 See, R. Chisholm, Person and Object, (LaSalle, Illinois: Open Court, 1976), esp. $23-46$ and $89-108$. 
memory theory, there simply is no reason to prefer his theory of personal identity over the two just mentioned. Nevertheless, the enormous appeal of the memory theory suggests that while Perry's attempt to identify the Mrelation is mistaken or question begging, perhaps there is some other more useful way that the relation can be identified and its nature elucidated. ${ }^{19}$ It is to such an alternative that I shall now turn.

Martin and Deutscher have argued that the appropriate sort of causal connection between a witnessing and a representing can be defined only be recourse to the notion of a memory trace. Once we recognise this, then the M-relation can be identified as the relation that obtains when a witnessing leaves a trace on the brain which trace persists and is later the cause of an ostensible consciousness of a past event. Thus, the M-relation is identified with the causal mechanisms, discoverable by scientists, that occur when we really remember. But does such a manuever really avoid circularity? I do not believe so. For, it may be asked, how are these causal mechanisms to be identified? How is the scientist to know what relation to look for between a witnessing and a representing unless he is first able to establish that the ostensible rememberer is really remembering? In other words, our knowledge that a certain person is really remembering a past event, must be prior to our knowledge of the causal processes involved, for if we were not able to distinguish real memories from apparent memories before we examined their physiological and neurological correlates, then we would not know which causal processes to identify with real memory. Thus, we cannot hope to understand real memory in terms of these causal processes. Of course, it might be argued that the characteristic process involved in real memory can be identified without an appeal to real memeory, by an appeal to personal identity. That is, one first determines cases of personal identity and then by uncovering the physical mechanisms that lead from the witnessing to the representing in those cases, one arrives at cases of real memory. Whatever advantages that has as an analysis of memory, clearly it is utterly useless in our attempt to understand the nature of personal identity.

In another paper Perry attempts to give an analysis of personal identity that appeals to a wider kind of "appropriate" causal relationship than the Mrelation. Concerning this relation Perry says:

This new relation, under the description, the relation that explains (or, if known, would explain) the approximate validity of the principles about humans we subscribe to, is my candidate for the analysis of personal

19 It has even been argued that Locke held a causal theory of memory and personal identity. See, M.W. Hughes "Personal Identity: A Defense of Locke", Philosophy 50 No. 192 (April 1975), 184-186. And A. Flew, although he disagrees that Locke held a causal theory believes that "there is much to be said for 'a causal theory', considered in its own right". A. Flew, "Personal Identity: Offences of the Hughes Defence", Philo. sophy 52 No. 200 (April, 1977), 206. 
identity. That is, it is the unity relation for persons, that relation which obtains between two stages if and only if there is a person of which both are stages. I shall call this the P-relation. ${ }^{20}$

The P-relation is the causal relation that both explains and unites the similar and continuous psychological characteristics that are exhibited by one and the same person. Thus, personal identity is analysed in terms of psychological continuity that is caused in the characteristic way. ${ }^{21}$ But what is the "characteristic way" in which psychological continuity is caused? What is the Prelation? Perry's answer is that "If the assumptions about the role of the brain made by recent philosophers is correct, the relation of having the same brain is at least a promising candidate for the P-relation". ${ }^{22}$ Although perhaps a promising candidate, the P-relation cannot be identifed with having the same brain because a person can retain her or his personal identity - be who they are to themselves and to others - even if their psychological continuity is not caused by the same brain, that is, even if a structurally similar brain replaces the original brain. Thus, either the P-relation cannot be identified with having the same brain or if it is, then the P-relation is not necessary for personal identity. Furthermore, the P-relation is not sufficient for personal identity either, for it is possible to have stages associated with one brain that are not stages of one and the same person. Thus, if having the same brain is the P-relation, then the P-relation is neither necessary nor sufficient for personal identity, and consequently its introduction is useless in answering questions surrounding the topic of personal identity.

In response to the above objections, it is open to the causal theorist to simply deny the possibilities I have mentioned. That is, the causal theorist who identifies the P-relation with having the same brain may claim that cases where we do not have literally the same brain and do have personal identity, and cases where we do have the same brain, but do not have personal identity are impossible, contradictory cases. Ultimately, such a view does not, however, avoid the problem of circularity. For in order to determine which experiences are experiences that belong to me, I must determine which experiences are related to the same brain. But it is not enough that they all be experiences related to the same brain, for in order for them to be mine they must be related to $m y$ brain and now we must ask which brain is $m y$ brain? The point

20 J. Perry, "The Importance of Being Identical", op. cit., 71; emphasis added.

21 In his article "Lewis, Perry, and What Matters", op. cit., D. Parfit expresses his close agreement with Perry on personal identity:

Perry's view is that a person at one time and a person at another time are the same person if they stand in the relation which normally causes psychological continuity (p. 71). My view was that they are the same person if they stand in the relation of psychological continuity, my account of which required a normal cause. So we agree that there is personal identity when there is both psychological continuity and its normal cause $(101-102)$. 
here is that unless we first can determine and understand what it is for an experience to be mine, or for two experiences to belong to the same person, we will not be able to determine that an experience belongs to my brain, for we will not know which brain is my brain. In still other words, it appears that the only answer to the question which brain is my brain is the circular one that my brain is the brain that is related in the characteristic way to those experiences that are experiences of one and the same person, namely me. Thus, the P-relation qua having the same brain does not elucidate the notion of personal identity, it presupposes it.

The causal theories of memory and personal identity have an initial plausibility because they appear to provide the link that enables us to distinguish cases of real and apparent memory and cases of real and apparent personal identity. Here as elsewhere, however, appearances are deceiving. The "characteristic" causal connection is never clearly identified and so becomes a something we know not what that fails to serve the purpose for which it was introduced. The appeal to characteristic causal relations have become standard addition to many recent analyses of such concepts as perception, knowledge, emotion, and action. ${ }^{23}$ If my critique of the causal theory of personal identity is correct, however, then it would appear to be applicable to any analysis that attempts to understand the nature of a concept by an appeal to a characteristic causal relation. To establish this in detail, with respect to each of the above mentioned concepts, is beyond the scope of this paper. ${ }^{24}$

\section{UNIVERSITY OF MICHIGAN-FLINT \\ FLINT, MI 48503 USA}

23 See, A.I. Goldman, A Theory of Human Action, (Englewood Clifts, New Jersey: Prentice-Hall, 1970), 56-63. H.P. Grice, "The Causal Theory of Perception", reprinted in Perceiving, Sensing, and Knowing, R. Swartz (ed.), 463. A.I. Goldman, 'A Causal Theory of Knowing', in Knowing, M.D. Roth and L. Galis (eds.) (New York: Random House, 1970), 83. R.M. Gordon, "The Aboutness of Emotions", American Philosophical Quarterly, Vol. 11 No. 1 (January 1974), 27-36.

A slightly shorter version of this paper was read at the Pacific Division Meetings of the American Philosophical Association in Portland, Oregon on March 26, 1981. I have benefited from discussions with Charles E.M. Dunlop. 